\title{
Syn-Selective anti-Markovnikov Intramolecular Hydroamination
}

Metal-Catalyzed Asymmetric Synthesis and Stereoselective

Reactions

\section{Key words}

rhodium(I)

anti-Markovnikov

hydroamination
Significance: Using a Rh(I)/DPPB catalyst system, aminoolefins undergo a remarkably selective anti-Markovnikov hydroamination reaction to generate 3-arylpiperidines in good yields. When the aminoolefin is appropriately substituted $(R \neq H)$, products are obtained with high degrees of synselectivity; presumably due to equatorial placement of the substituents in a chair-like transition state. The $\mathrm{N}$-methyl substituent is necessary for the reaction to proceed; however, this group can be easily cleaved (see scheme).
Comment: Hydroamination reactions have been studied for decades, with many advances improving the efficiency of the reaction. However, the main limitation of hydroamination is a lack of Markovnikov/anti-Markovnikov selectivity (see review below), which is overcome using the rhodium catalyst system reported. The products of this reaction are medicinally interesting, as 3-arylpiperidines have found activity as dopamine autoreceptor antagonists which stimulate dopamine turnover without inducing hypoactivity. Development of an enantioselective anti-Markovnikov hydroamination would provide rapid access to this class of bioactive compounds.

Review: S. Hong, T. J. Marks Acc. Chem. Res. 2004, 37, 673-686. 